\title{
A NEW UNSUPERVISED CHANGE DETECTION APPROACH BASED ON DWT IMAGE FUSION AND BACKTRACKING SEARCH OPTIMIZATION ALGORITHM FOR OPTICAL REMOTE SENSING DATA
}

\author{
U. H. Atasever ${ }^{\text {a, } *, \text { P. Civicioglu }}{ }^{\text {b }}$ E. Besdok ${ }^{\mathrm{a}}$, C. Ozkan ${ }^{\mathrm{a}}$ \\ ${ }^{a}$ Department of Geomatic Engineering, Erciyes University, Kayseri, Turkey \\ (uhatasever, cozkan, ebesdok)@erciyes.edu.tr \\ ${ }^{b}$ College of Aviation, Dept. of Aircraft Electrics and Electronics, Erciyes University, Kayseri, Turkey \\ civici@erciyes.edu.tr
}

Commission VI, WG VI/4

KEY WORDS: Change Detection, DWT Based Image Fusion, Backtracking Search Optimization Algorithm (BSA)

\begin{abstract}
:
Change detection is one of the most important subjects of remote sensing discipline. In this paper, a new unsupervised change detection approach is proposed for multi-temporal remotely sensed optic imagery. This approach does not require any prior information about changed and unchanged pixels. The approach is based on Discrete Wavelet Transform (DWT) based image fusion and Backtracking Search Optimization Algorithm (BSA). In the first step of the approach, absolute-valued difference image and absolute-valued log-ratio image is calculated from co-registered and radiometrically corrected multi-temporal images. Then, these difference images are fused using DWT. The fused image is filtered by median filter for edge information preservation and by wiener filter for image smoothing. Then, a min-max normalization is applied to the filtered data. The normalized data is clustered into two groups with BSA as changed and unchanged pixels by minimizing an objective function, unlike classical methods using CVA, PCA, FCM or K-means techniques. To show effectiveness of proposed approach, two remote sensing data sets, Sardinia and Mexico, are used. False Alarm, Missed Alarm, Total Alarm and Total Error Rate are selected as performance criteria to evaluate the effectiveness of new approach using ground truth images. Experimental results show that proposed approach is effective for unsupervised change detection of optical remote sensing data.
\end{abstract}

\section{INTRODUCTION}

The detection of changes on earth surface is one of the most important subjects of remote sensing discipline. In many applications (monitoring urban growth, disaster management, identifying the spatial difference after a forest fire, etc.), change detection based on remote sensing is vital to extract meaningful information (Cihlar et al 1992, Gopal and Woodcock 1996). Change detection is separated into two main topics as supervised and unsupervised. But in many applications, unsupervised change detection techniques are preferred due to not need any training data. Basic approaches used in change detection are change vector analysis (CVA), image differencing, principal component analysis (PCA) and image rationing. Among them, image differencing is the most common used approach in real world problems (Mishra et al. 2012). Over the last years, many unsupervised change detection methods based on differencing image have been improved such as PCAKmeans (Celik 2009), PCA-FCM, HCM Based (Ghosh et al. 2011), G-HCM Based (Ghosh et al. 2011), EM+MRF Based (Bruzzone and Prieto, 2000).

In some of the change detection methods, firstly, feature space is calculated from the multi-temporal images with the help of image differencing and then this feature space is divided into two clusters as changed and unchanged (Ghosh et al. 2011). In this step, performance of the clustering algorithm is very important. Over the last decade, artificial intelligence (AI) optimization algorithms have been tested for clustering. The studies show that these algorithms are more successful than the classical clustering techniques such as K-means, Fuzzy Cmeans (Zou et al. 2010, Karaboga and Ozturk 2011). In this study, a new artificial intelligence optimization algorithm, BSA, has been preferred for clustering in this approach.

In this study, a new automatic change detection approach based on DWT image fusion and BSA clustering for optic images is proposed. Experimental results prove that proposed approach is effective for unsupervised change detection.

\section{BACKTRACKING SEARCH OPTIMIZATION ALGORITHM (BSA)}

Optimization is one of the important research area in engineering problems and applied mathematics (Civicioglu, 2013). Optimization algorithms are tried to find best values for a system or model parameters in different conditions. Especially, image processing applications (De Falco et al. 2008), mechanical design or sensor displacement problems are most commonly applied problem types of optimization algorithms. In recent years, many artificial intelligence (AI) optimization algorithms are improved. Most commonly used algorithms are Differential Evolution (DE), Artificial Bee Colony (ABC), Genetic and Particle Swarm Optimization Algorithm (PSO) (Civicioglu, 2013). But in this study, a new AI algorithm, BSA, is preferred due to robustness and

* Corresponding author, uhatasever@erciyes.edu.tr, +90 352 2076666-32678 
effectiveness. BSA outperformed many algorithms such as $\mathrm{ABC}, \mathrm{DE}$ in many benchmark tests and real world problems (Civicioglu, 2013).

BSA is a population-based iterative optimization algorithm. BSA has five basic steps: Selection-1, Mutation, Crossover and Selection-2. Unlike many AI algorithms, BSA has single control parameter. Also, BSA has a very simple and strong structure which can be adapted to different numerical optimization problems (Civicioglu, 2013). BSA's strategies for generating trial populations have powerful exploration capabilities

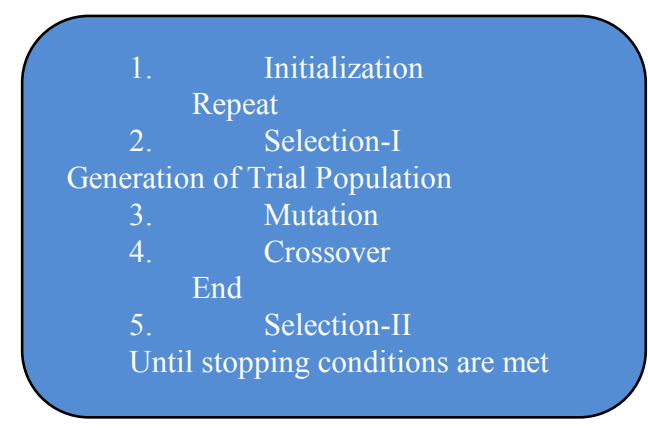

Table.1 General Structure of BSA

\section{CHANGE DETECTION BASED ON DWT IMAGE FUSION AND BSA CLUSTERING}

Firstly, two multi-temporal images must be registered to each other and if it is necessary the radiometric distortions must be corrected. Secondly, absolute-valued difference image and absolute-valued log-ratio image are calculated with equation (1) and equation (2), respectively:

$$
\begin{gathered}
X_{D}=\left|X_{T 2}-X_{T 1}\right| \\
X_{L R}=\left|\log \frac{X_{T 2}+1}{X_{T 2}+1}\right|
\end{gathered}
$$

In equation (1) and (2), $X_{D}$ is absolute-valued difference image; $X_{L R}$ is absolute-valued log-ratio image; $X_{T 1}$ and $X_{T 2}$ are multitemporal images (Celik, 2010).

In the third step, absolute-valued difference image and absolutevalued log-ratio image are fused with Discrete Wavelet Transformation (DWT). In DWT image fusion, Discrete Wavelet Transformation is applied to $X_{D}$ and $X_{L R}$ images and wavelet coefficients of each image are calculated. After that, combined wavelet coefficients are calculated by combination rules such as min, max or rand (Yingchun et al. 2011). Lastly, fused image $\left(X_{F u s}\right)$ is obtained by applying Inverse Discrete Wavelet Transformation (IDWT) to combined wavelet coefficients. In this step, $d b 8$ for decomposition, mean for approximations and $\min$ for details have been used.

In the fourth step, 2-D median filter with $3 \times 3$ window sizes and 2-D wiener filter with $11 \times 11$ window sizes are applied to $X_{F u s}$, respectively. Median filter has been used to preserve edge information and to suppress the isolated pixels in $X_{F u s}$. Wiener filter has been used for smoothing of $X_{F u s}$ without losing edge information.
After that, filtered data is normalized into $[0,1]$ interval in order to boost the performance of BSA. This technique, called also min-max normalization, is performed according to the equation (3):

$$
P_{\text {new }}=\frac{P-P_{\min }}{P_{\max }-P_{\min }}
$$

In equation (3), $P_{n e w}$ is the normalized value; $P, P_{\min }$ and $P_{\max }$ are the original value, minimum value and maximum value in the filtered data, respectively.

In the last step, normalized data is clustered into two classes by using BSA algorithm. Also, BSA needs an objective function for clustering. The objective function is given in equation (4) and (5):

$$
\begin{gathered}
d_{j}=\left\{x_{i} ; \min \left(\| x_{i}-\text { cent }_{j} \|_{2}\right\}\right. \\
\underset{\text { cent }_{j}}{\arg \min } \sum \| d_{j}-\text { cent }_{j} \|_{2}
\end{gathered}
$$

In equation (4) and (5), \|\|$_{2}$ is Euclidean distance, $x_{i}$ is $i^{\text {th }}$ pixel; cent ${ }_{j}$ is the centre of the $j^{\text {th }}$ class; $d_{j}$ represents all of the pixels in the $j^{\text {th }}$ class. As can be seen in the objective function, BSA optimizes the cluster centres so that these centres provide the least minimum total distance between the cluster centres and the pixels of each cluster in each iteration. General scheme of proposed approach is shown in figure 1.

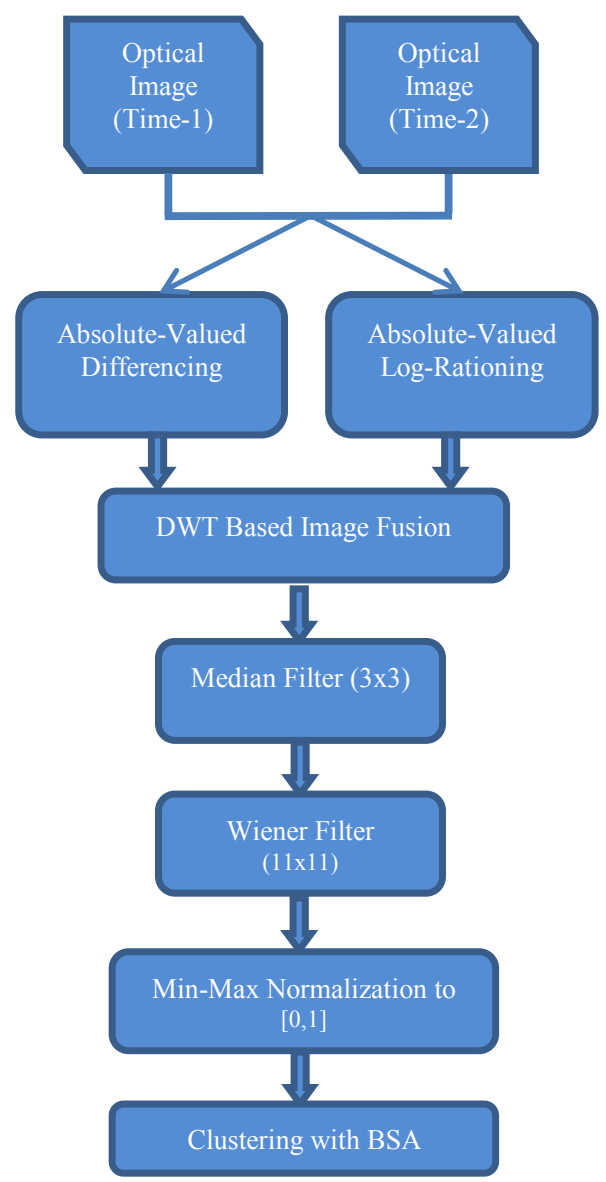

Figure 1. General scheme of proposed approach 


\section{DATASETS AND EXPERIMENTAL RESULTS}

In this study, both Sardinia dataset (Landsat 5 TM) set and Mexico data set (Landsat 7 ETM) are used. Sardinia data set belongs to Mulargia Lake in Sardinia Island from September 1995 and September 1996 years. The image sizes are 300x412 pixels. Mexico data set belongs to an area in Mexico from April 2000 and May 2002 years. The image sizes are 512x512 pixels. Because the histograms of multi-temporal Landsat images are very similar to each other, any radiometric correction is not applied. The fourth bands of the multispectral images are selected for change detection process because of the reflectance characteristics of water, land and vegetation objects (Hao et al. 2013). Moreover, a ground truth map is employed to calculate the performance Sardinia dataset and Mexico dataset are shown in figure 2 (Ghosh et al. 2011).

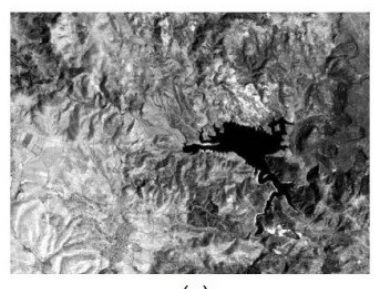

(a)

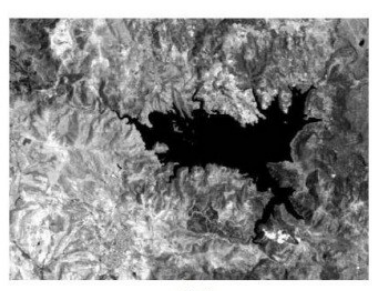

(b)

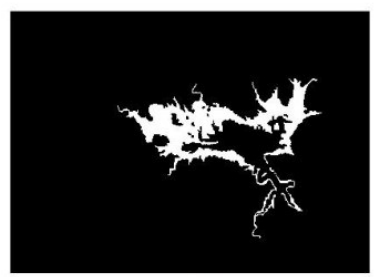

(c)

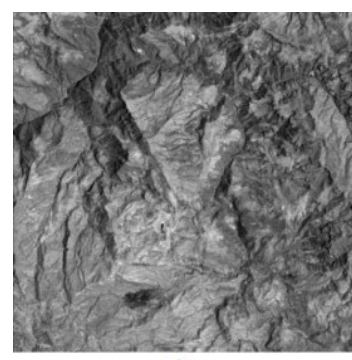

(d)

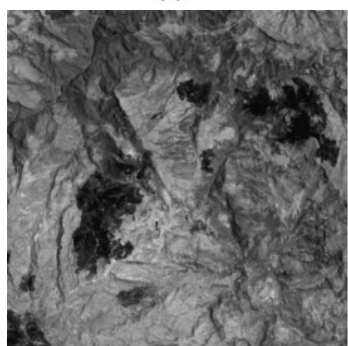

(e)

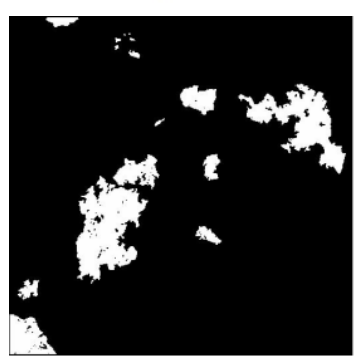

(f)
Figure 2. (a)Sardinia September 1995, (b) Sardinia September 1996, (c) Sardinia dataset ground truth map, (d) Mexico April 2000, (e) Mexico May 2002, (f) Mexico dataset ground truth

$$
\text { map }
$$

The measures below are used for quantitative analysis of proposed approach:

-False Alarm (FA): Number of unchanged pixels identified as changed pixels.

-Missed Alarm (MA): Number of changed pixels identified as unchanged pixels.

-Total Error (TE): Total number of incorrectly categorized, which is sum of FA and MA.

-Total Error Rate (TER): Ratio of sum of FA and MA to number of image pixels $(\mathrm{PN}) . T E R=(F A+M A) /(P N) \times 100$
In the application of Sardinia dataset and Mexico dataset, proposed approach is compared with HCM, G-HCM, PCAFCM and PCA-K Means methods. Except PCA-K Means and PCA-FCM, results of the other methods are taken from the original papers cited in table 2 and table 3 . Thus, the efficiency of the proposed approach is analyzed for optical remote sensing images. The visual results and error values obtained from ground truth map data are given in figure 3 , figure 4 and table 2 , table 3, respectively. As seen from table 2, proposed approach gets the best change detection accuracy in all methods for Sardinia dataset. Preferred scale factor is an inverse random number coming from a Normal distribution with 0 mean and standard deviation of 5 . Population and generation number values of BSA are 10 and 100, respectively. These parameters are also have been used for Mexico dataset.

As seen from figure 4 and table 3, proposed approach can generate quite successful results, i.e. Except G-HCM method, the proposed approach outperforms other change detection methods for Mexico dataset.

\begin{tabular}{cclcc}
\hline Methods Used & $\begin{array}{c}\text { False } \\
\text { Alarm }\end{array}$ & $\begin{array}{c}\text { Missed } \\
\text { Alarm }\end{array}$ & $\begin{array}{c}\text { Total } \\
\text { Error }\end{array}$ & $\begin{array}{c}\text { Total } \\
\text { Error } \\
\text { Rate }\end{array}$ \\
\hline PCA-FCM & 1008 & 3729 & 4737 & 1.80 \\
PCA-Kmeans & 1024 & 3662 & 4686 & 1.78 \\
HCM & 3425 & 747 & 4172 & 1.59 \\
(Ghosh et al. 2011) & & & & \\
Proposed & $\mathbf{1 1 4 0}$ & $\mathbf{2 6 2 5}$ & $\mathbf{3 7 6 5}$ & $\mathbf{1 . 4 3}$ \\
$\begin{array}{c}\text { G-HCM } \\
\text { (Ghosh et al. 2011) }\end{array}$ & 2827 & 785 & 3612 & 1.38 \\
\hline
\end{tabular}

Table 2. The quantitative results of Mexico Landsat 7 image data set.

\begin{tabular}{ccccc}
\hline Methods Used & $\begin{array}{c}\text { False } \\
\text { Alarm }\end{array}$ & $\begin{array}{c}\text { Missed } \\
\text { Alarm }\end{array}$ & $\begin{array}{c}\text { Total } \\
\text { Error }\end{array}$ & $\begin{array}{c}\text { Total } \\
\text { Error } \\
\text { Rate }\end{array}$ \\
\hline $\begin{array}{c}\text { HCM } \\
\text { (Ghosh et al. 2011) }\end{array}$ & 275 & 4133 & 4408 & 3.56 \\
$\begin{array}{c}\text { G-HCM } \\
\text { (Ghosh et al. 2011) }\end{array}$ & 3006 & 132 & 3138 & 2.53 \\
PCA-FCM & 1855 & 752 & 2607 & 2.10 \\
PCA-Kmeans & 1603 & 832 & 2435 & 1.97 \\
Proposed & $\mathbf{1 0 8 7}$ & $\mathbf{8 6 3}$ & $\mathbf{1 9 5 0}$ & $\mathbf{1 . 5 7}$ \\
\hline
\end{tabular}

Table 3. The quantitative results of Sardinia Landsat 5 image data set.

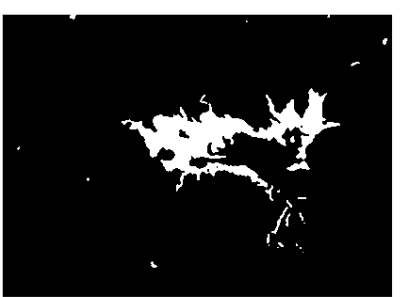

(a)

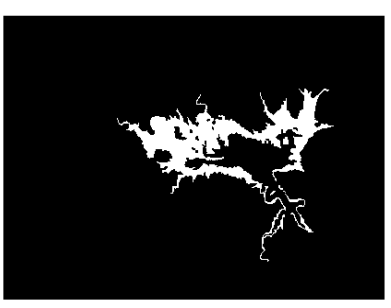

(b)
Figure 3. Change detection result of Sardinia data set,

(a) Proposed approach, (b) Ground truth 


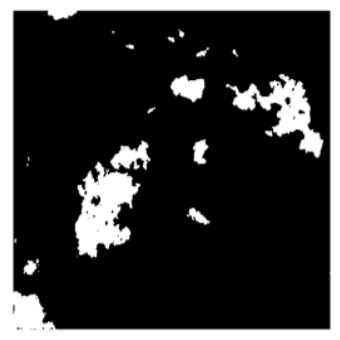

(a)

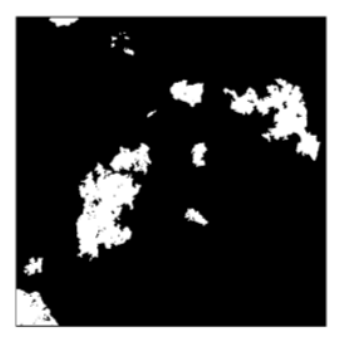

(b)
Figure 4. Change detection result of Mexico data set,

(a) Proposed approach, (b) Ground truth

\section{CONCLUSIONS}

In this paper, a new unsupervised change detection approach is proposed using DWT image fusion and BSA clustering for multi-temporal optical data. The performance of proposed approach is compared with a number of change detection techniques. The applications with Sardinia dataset (Landsat 5) and Mexico dataset (Landsat 7) show that the blending with DWT based image fusion and BSA based clustering make the approach more effective and successful. Considering the calculation time, the proposed approach is slower than other methods because it combines DWT and BSA.

\section{ACKNOWLEDGEMENTS}

This work was supported by The Scientific and Technological Council of Turkey (TUBITAK) under Grant [114Y049].

\section{REFERENCES}

Bruzzone, L. and D. Prieto. 2000. Automatic analysis of the difference image for unsupervised change detection. IEEE Transactions on Geoscience and Remote Sensing, 38(3), pp. $1171-1182$

Celik, T. 2009. Unsupervised Change Detection in Satellite Images Using Principal Component Analysis and k-Means Clustering. IEEE Transactions on Geoscience and Remote Sensing Letters, 6(4), pp. 772-776.

Celik, T. 2010. Change Detection in Satellite Images Using a Genetic Algorithm Approach. IEEE Geoscience and Remote Sensing Letters, 7(2), pp. 386-390.

Cihlar, J., T. Pultz., and A. L. Gray. 1992. Change Detection with Synthetic Aperture Radar. International Journal of Remote Sensing, 13, pp. 401-414

Civicioglu, P. 2012. Transforming Geocentric Cartesian Coordinates to Geodetic Coordinates by Using Differential Search Algorithm. Computers and Geosciences, 46, pp.229247.

De Falco, I., A. D. Cioppa, D. Maisto, and E. Tarantino. 2008. Differential Evolution as a viable tool for satellite image registration, Applied Soft Computing, 8(4), pp. 1453-1462.

Ghosh, A., N. S. Mishra, and S. Ghosh. 2011. Fuzzy Clustering Algorithms for Unsupervised Change Detection in Remote Sensing Images. Information Sciences, 181, pp. 699-715.
Gopal, S. and C. Woodcock. 1996. Remote Sensing Of Forest Change Using Artificial Neural Networks. IEEE Transactions on Geoscience and Remote Sensing, 34, pp.398-404.

Hao, M., H. Zhang, W. Z. Shi, and K. Z. Deng. 2013. Unsupervised change detection using fuzzy c-means and MRF from remotely sensed images. Remote Sensing Letters, 4(12), pp. 1185-1194.

Karaboga, D., and C. Ozturk. 2011. A novel clustering approach: Artificial Bee Colony (ABC) algorithm. Applied Soft Computing, 11(1), pp. 652-657.

Mishra, N. S., S. Ghosh, and A. Ghosh. 2012. Fuzzy clustering algorithms incorporating local information for change detection in remotely sensed images. Applied Soft Computing, 12, pp. 2683-2692.

Yingchun, T., Q. Yali, W. Hao, and W. Gang. 2011. Change Detection of Multi-temporal Remote Sensing Data Using Wavelet-Based Fusion and K-Means Clustering. Intelligent Human-Machine Systems and Cybernetics (IHMSC), 2011 International Conference on, pp. 119-122.

Zou, W., Y. Zhu, H. Chen, X. Sui. 2010. A Clustering Approach Using Cooperative Artificial Bee Colony Algorithm. Discrete Dynamics in Nature and Society, vol .2010. 